\title{
Oxygen Abundance and Stellar Populations in the Three Most Metal-Deficient Emission-Line Galaxies Known in the Local Universe: SBS 0335-052 E, SBS 0335-052 $\mathrm{W}$ and $\mathrm{I} \mathrm{Zw} 18$
}

\author{
P. Papaderos ${ }^{1}$, T. Yakobchuk ${ }^{2}$, Y. I. Izotov $^{2}$, N. G. Guseva ${ }^{2}$, \\ and K. J. Fricke ${ }^{1}$ \\ ${ }^{1}$ Institute for Astrophysics, Friedrich-Hund-Platz 1, 37077 Göttingen, Germany \\ ${ }^{2}$ Main Astronomical Observatory, Zabolotnoho 27, Kyiv 03680, Ukraine
}

We present new spectroscopic observations of the system of the two blue compact dwarf (BCD) galaxies SBS 0335-052 W and SBS 0335-052 E with the 3.6m ESO telescope. The oxygen abundance in SBS 0335-052 W was determined to be $12+\log (\mathrm{O} / \mathrm{H})=7.13 \pm 0.08$, confirming that this galaxy is the most metal-deficient emission-line galaxy known. We find that the oxygen abundance in SBS 0335-052 E varies from region to region in the range from 7.20 to 7.31, suggesting the presence of an abundance gradient over a spatial scale of $\sim 1 \mathrm{kpc}$. Signatures of early carbon-type Wolf-Rayet stars are detected in cluster \#3 of SBS 0335-052 E, corresponding to the emission of 20 to 130 WC4 stars, depending on the adopted luminosity of a single WC4 star in the CIV $\lambda 4658$ emission line (Papaderos et al. 2006).

Aditionally, we study the evolutionary status of another extremely metal-deficient Wolf-Rayet galaxy, I Zw $18(12+\log (\mathrm{O} / \mathrm{H})=7.17 \ldots 7.22$; Thuan \& Izotov 2005), based on the analysis of deep HST ACS data with the point-source photometry package DOLPHOT. Our goal is to study the oldest stellar populations in this BCD and search for red giant branch (RGB) stars. A detailed analysis of the derived color-magnitude diagrams (CMDs), that reach $\sim 29 \mathrm{mag}$ in $V$ and $I$, has led us to the following conclusions (Yakobchuk \& Izotov 2006, Yakobchuk et al. 2006):

i) there is no substantial population of RGB stars in the CMDs of I Zw 18's main body and $\mathrm{C}$ component. Probable RGB stars are hard to distinguish from the asymptotic giant branch $(\mathrm{AGB})$ stars and have very blue colors $(V-I \approx 1.05 \mathrm{mag}$ in the main body) for the given metallicity of $Z=0.0004$. ii) the population of $R G B$ stars is not dominant in the CMD of the halo of I Zw 18, implying that all stars in I Zw 18 are quite young and have not yet diffused over a large volume. I $\mathrm{Zw} 18$ appears therefore to be the only known galaxy without a halo of old stars. iii) the apparent magnitudes of the tip of the red giant branch (TRGB) for the main body and the C component of I $\mathrm{Zw} 18$ differ by 0.7 mag. Using the theoretical dependences of the $M_{I}$ (TRGB) on the age and the color (Girardi et al. 2000), this contradiction can be resolved if we assume that the formation of the RGB stars in I Zw 18 is not yet finished and the red giants have different ages in the main body and the $\mathrm{C}$ component.

Girardi et al. 2000, A\&AS, 141, 371 - Papaderos et al. 2006, A\&A, 454, 119 - Thuan, T.X. \& Izotov, Y.I. 2005, ApJS, 161, 240 · Yakobchuk T. \& Izotov Y.I. 2006, Kinematics and Physics of Celestial Bodies, in press. · Yakobchuk, T. et al. 2006, in prep.

This work has been supported by Deutsche Forschungsgemeinschaft grants 436 UKR 17/25/05 and PA 1228/5-1. 